\section{Was Wrong on "Monophyletic"}

\author{
S. Joshua Swamidass (D) \\ https://doi.org/10.54739/jyc3
}

was wrong. I incorrectly used the terms "monophyletic" and "polyphyletic" in my book, The Genealogical Adam and Eve. My intended meaning was clear from context, but departed far from the usual meaning of the words. As biologist Richard Buggs notes in his review of my book:

\section{Confusingly, Swamidass describes his created Adam and Eve as "monophyletic" with evolved human beings, but by this he means "of the same biological type" (p. 85), rather than the more usual definition of "sharing common ancestry".}

Dr. Buggs is entirely correct. I was wrong. Devin Gouvêa and Kenneth Kemp, two philosophers, were also quick to identify this error and I am grateful that they brought this to my attention. This is an error that needs to be corrected.

\section{Monophylogeny and Polyphylogeny}

Before I explain the error on monophyletic and polyphyletic (the "phyletic" terms), I want to make a distinction from monophylogeny and polyphylogeny, the "-phylogeny" terms.

There was also debate about my use of the "-phylogeny" terms, but they are not common within scientific discourse. For this reason, I am continuing to use monophylogeny to mean "of the same biological type" and polyphylogeny to mean "of different biological types." In context, I am using these terms to refer to biological hummaness and race.

This brings us to another issue too important to gloss: what is a biological type? The notion of biological type and species, like many concepts, is multivalent in biology with many meanings in different contexts. The Stanford Encyclopedia of Philosophy expounds the many ways "species" is understood in biology. Jodie Hey gives over

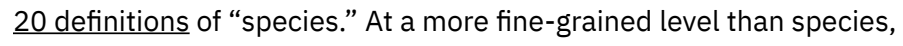
there are 10 s of thousands of papers studying biologically distinct cancer types and subtypes. So what biologists mean by "biological type" is very fluid. In the context of the book, what I mean by monophylogeny is that we are all equally human, with the same potential.

Even if humans (in one narrow and technical sense) arise by genealogical descent from Adam and Eve, there was full humanness outside the garden. Therefore, humanness does not spread by genealogical descent. If Adam and Eve were recent, that would not make much sense to me. Now, if Adam and Eve were ancient, that changes the range of possibilities, but those possibilities are out of scope of the The Genealogical Adam and Eve.

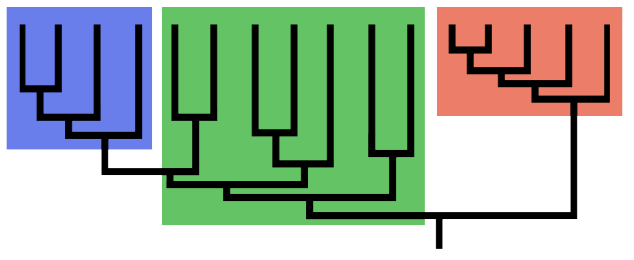

To understand more about the scientific evidence that we are all equally human, I recommend the new book by Adam Rutherford, How to Argue with A Racist and Gene Olson's Mapping Human History. Perhaps I will write more about this soon too. By affirming monophylogeny. I am referencing this work, affirming the biological unity of all humankind, affirming we are all equally human with equal humanness.

\section{Monophyletic and Polyphyletic}

So, what did I get wrong? I misused the "-phyletic" terms.

My use of the term monophyletic in the book was intended to mean "of the same species or subspecies." This is not what the term actually means. Instead, it means a group of different species that together are all the descendent species of common ancestor. For example, the figure shows in yellow that simians are all monophyletic. Likewise, chimpanzees and humans are monophyletic, even though they are different species. My intended meaning here is close to the term conspecific, of the same species, and several edits replace monophyletic with this term.

My use of the term polyphyletic in the book was intended to mean "of different species or subspecies." This is not what the term actually means. Instead, it means a group of different species that arise from different lineages. For example, the figure shows in red that nightactive primates are polyphyletic.

The good news is that my intended meaning is clear in context, even though it is in conflict with the correct definitions of the terms. The common story of racist theories of origins is that humans today are of different biological types. These biological differences arise due to some sort of separation in the past. That message remains clear through the book, even though I incorrectly used the terms monophyletic and polyphyletic.

The bad news is that several corrections are required to fix this error. I hope that this correction, especially because it is made early in the life of the book, will head off misunderstanding. There were several errors on where I lay out the definitions of polygenesis (p. 120). Here is the rewrite, which can be quoted when needed in place of the text in the book,

- Monophylogeny means a group is all the same biological type. Humans are conspecific, meaning that we are all the same species and subspecies.

Most the errors were here, on page 120, in these definitions. There are more corrections through the book, detailed in the errata. This 


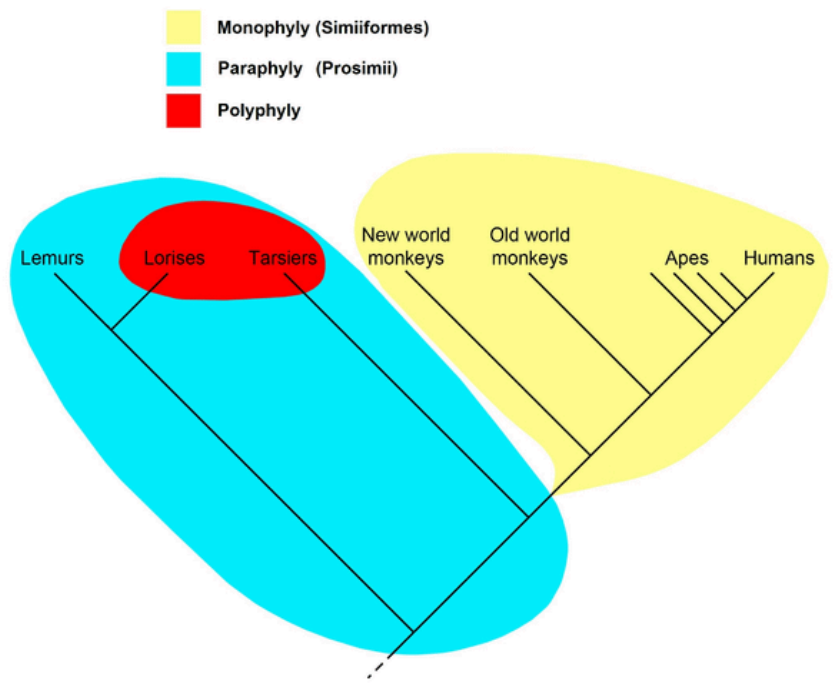

A cladogram of the <a href="https://en.wikipedia.org/wiki/Primate" target='_blank' rel='noopener nofollow' class='external-link' class='external-link' $>$ primates $\langle/ a\rangle$, showing a <em >monophyletic </em > taxon: <em >the simians $</$ em $>$ (in yellow); a $<$ em $>$ paraphyletic $</$ em $>$ taxon: $<$ em $>$ the prosimians $</$ em $>$ (in cyan, including the red patch); and a <em >polyphyletic </em > group: <em $>$ the night-active primates, i.e., the</em><a href="https://en.wikipedia.org/wiki/Loris" target='_blank' rel='noopener nofollow' class='external-link' class='external-

link' $><$ em $>$ lorises $</$ em $></$ a $><$ em $>$ and the $</$ em $><$ a href="https://en.wikipedia.org/ wiki/Tarsier" target='_blank' rel='noopener nofollow' class='external-link' class='external-link' $><$ em $>$ tarsiers $</$ em $></ a>$ (in red). Credit: $<$ a

href="https://en.wikipedia.org/wiki/Monophyly" target='_blank' rel='noopener nofollow' class='external-link' class='external-link' $>$ Wikipedia $\langle/ a>$.

\section{References}

https://peacefulscience.org/books/genealogical-adam-eve/

https://natureecoevocommunity.nature.com/users/24561-richardbuggs/posts/61200-adam-and-eve-our-ghostly-ancestors

https://plato.stanford.edu/entries/species

https://www.nature.com/scitable/topicpage/why-should-we-careabout-species-4277923

https://www.ncbi.nlm.nih.gov/pubmed/?term=cancer+subtype

https://peacefulscience.org/books/argue-with-racist/ post should clear the record. Hopefully these changes will be made in future printing runs.

I acknowledge philosophers Devin Gouvêa, Alan Love, and Kenneth Kemp and biologist Jeffery Schloss for reviewing this correction. I am particularly grateful to Devin Gouvêa, Kenneth Kemp, and Richard Buggs for bringing this error to my attention. https://peacefulscience.org/books/mapping-human-history/ https://en.wikipedia.org/wiki/Primate https://en.wikipedia.org/wiki/Loris https://en.wikipedia.org/wiki/Tarsier https://en.wikipedia.org/wiki/Monophyly https://peacefulscience.org/pdf/genealogical-adam-eve-erratajan2021.pdf 